\title{
THE HERMITE POLYNOMIALS AND THE BESSEL FUNCTIONS FROM A GENERAL POINT OF VIEW
}

\author{
G. DATTOLI, H. M. SRIVASTAVA, and D. SACCHETTI
}

Received 5 November 2002

\begin{abstract}
We introduce new families of Hermite polynomials and of Bessel functions from a point of view involving the use of nonexponential generating functions. We study their relevant recurrence relations and show that they satisfy differentialdifference equations which are isospectral to those of the ordinary case. We also indicate the usefulness of some of these new families.
\end{abstract}

2000 Mathematics Subject Classification: 33C10, 33C45, 33C50.

1. Introduction. We consider a continuous and infinitely differentiable function $f(x)$ and associate with it the following generating function:

$$
f\left(x t+y t^{2}\right)=\sum_{n=0}^{\infty} \frac{t^{n}}{n !} \phi_{n}(x, y),
$$

where $\phi_{n}(x, y)$ are the two-variable polynomials which will be shown to be a suitable generalization of the Hermite-Kampé de Fériet (HKdF) family [1] or a particular case of the Boas-Buck polynomials [2]. As it is well known, the HKdF polynomials are generated by (1.1) when $f(x)$ reduces to an exponential function, while in the case of Boas-Buck polynomials, the argument of $f$ should be replaced by $x g(t)$ with

$$
g(t)=\sum_{n=0}^{\infty} g_{n} t^{n+1}
$$

Here, in this paper, we will consider the first aspect only, namely $\phi_{n}(x, y)$, as generalized forms of the HKdF polynomials. Within the same framework, we will also introduce some generalized forms of the Bessel functions.

Unlike the exponential, $f(x)$ does not, in general, possess any semigroup property for which

$$
f(x+y)=f(x) f(y)
$$

Yet we can make use of the procedure developed by Dattoli et al. [7], which allows the use of a formal semigroup property of umbral nature. 
By assuming that $f(x)$ can be expanded in series as follows:

$$
f(x)=\sum_{s=0}^{\infty} \frac{f_{s}}{s !} x^{s},
$$

we introduce the operator $\hat{f}$ defined in such a way that

$$
(\hat{f})^{s}=f_{s}, \quad(\hat{f})^{r} f_{s}=f_{s+r} .
$$

Thus, we can write

$$
f(x)=\exp [(\hat{f} x)]=\sum_{s=0}^{\infty} \frac{(\hat{f} x)^{s}}{s !}=\sum_{s=0}^{\infty} \frac{f_{s}}{s !} x^{s} .
$$

Within the context of such a formalism, the semigroup property of the exponential function can be replaced by

$$
\begin{aligned}
f(x+y) & =\exp [\hat{f}(x+y)] \\
& =\exp [(\hat{f} x)+(\hat{f} y)] \\
& =\exp [(\hat{f} x)] \exp [(\hat{f} y)] .
\end{aligned}
$$

The usefulness of (1.7) will be observed in treating directly the generating function (1.1) in order to derive the explicit form of the polynomials $\phi_{n}(x, y)$.

Making use of (1.5), (1.6), and (1.7), we can conclude that

$$
\begin{aligned}
f\left(x t+y t^{2}\right) & =\exp [(\hat{f} x t)] \exp \left[\left(\hat{f} y t^{2}\right)\right] \\
& =\sum_{r=0}^{\infty} \frac{(\hat{f} x)^{r} t^{r}}{r !} \sum_{s=0}^{\infty} \frac{(\hat{f} y)^{s} t^{2 s}}{s !} .
\end{aligned}
$$

Upon setting $r+2 s=n$ in (1.8), if we apply the second equation in (1.5) and then equate the coefficients of $t^{n}$ from both sides of the resulting equation, we obtain

$$
\phi_{n}(x, y)=n ! \sum_{s=0}^{[n / 2]} \frac{f_{n-s} x^{n-2 s} y^{s}}{(n-2 s) ! s !}
$$

which reduces to the standard HKdF form for $f_{n}=1$ (see [1]).

Various properties and possible generalizations of the above polynomials will be discussed in the following sections, where we will also consider the possibility of developing an approach to the theory of a new form of Bessel-like functions, which can be developed from nonexponential generating functions.

2. The neo-Hermite polynomials $\phi_{n}(x, y)$. In this section, we aim at developing the relevant theory of what we call the neo-Hermite polynomials by 
means of that in the ordinary case. By applying the properties of the $\hat{f}$ operator, it is quite straightforward to prove that $\phi_{n}(x, y)$ satisfies the following recurrences relations:

$$
\begin{gathered}
\hat{f}^{-1} \frac{\partial}{\partial x} \phi_{n}(x, y)=n \phi_{n-1}(x, y) \\
{\left[(\hat{f} x)+2 y \frac{\partial}{\partial x}\right] \phi_{n}(x, y)=\phi_{n+1}(x, y) .}
\end{gathered}
$$

Thus, accordingly, it is easily seen that they satisfy the differential equation

$$
\left[2 y \hat{f}^{-1} \frac{\partial^{2}}{\partial x^{2}}+x \frac{\partial}{\partial x}\right] \phi_{n}(x, y)=n \phi_{n}(x, y) .
$$

From the recurrence relation (2.2), it also follows that

$$
\phi_{n}(x, y)=\left[(\hat{f} x)+2 y \frac{\partial}{\partial x}\right]^{n} f_{0}
$$

More generally, we can state the following Burchnall-type operational relation (see, e.g., [5]):

$$
\left[(\hat{f} x)+2 y \frac{\partial}{\partial x}\right]^{n}=\sum_{s=0}^{n}(2 y)^{s}\left(\begin{array}{l}
n \\
s
\end{array}\right) \phi_{n-s}(x, y) \frac{\partial^{s}}{\partial x^{s}}
$$

In order to prove (2.5), we begin by observing that

$$
\sum_{n=0}^{\infty} \frac{t^{n}}{n !}\left[(\hat{f} x)+2 y \frac{\partial}{\partial x}\right]^{n}=\exp \left[(\hat{f} x t)+2 y t \frac{\partial}{\partial x}\right] .
$$

Now, by applying the Weyl operational rule (see [6]) for the noncommutative operators

$$
\hat{A}=(\hat{f} x t), \quad \widehat{B}=2 y t \frac{\partial}{\partial x}
$$

we find that

$$
[\hat{A}, \hat{B}]=-2\left(\hat{f} y t^{2}\right)
$$

so that we have

$$
\exp \left[(\hat{f} x t)+2 y t \frac{\partial}{\partial x}\right]=\exp \left[(\hat{f} x t)+\left(\hat{f} y t^{2}\right)\right] \cdot \exp \left[2 y t \frac{\partial}{\partial x}\right]
$$

which can easily be manipulated to get the Burchnall-type operational relation (2.5).

It is also important to emphasize that, by analogy with the ordinary HKdF polynomials, the polynomials $\phi_{n}(x, y)$ satisfy the following partial differential 
equation:

$$
\frac{\partial}{\partial y} \phi_{n}(x, y)=\hat{f}^{-1} \frac{\partial^{2}}{\partial x^{2}} \phi_{n}(x, y)
$$

with

$$
\phi_{n}(x, 0)=f_{n} x^{n} .
$$

Thus, the polynomials $\phi_{n}(x, y)$ can be constructed according to the following operational rule:

$$
\phi_{n}(x, y)=\exp \left[\left(\hat{f}^{-1} y \frac{\partial^{2}}{\partial x^{2}}\right)\right] f_{n} x^{n}
$$

whose validity can immediately be checked by using the rule stated in the introductory remarks.

The following observations are prompted by the results which we have presented in this paper so far.

(a) We can construct, from a given analytic function $f$, a family of polynomials which we will define as the umbral image of the ordinary HKdF polynomials.

(b) Most of the properties of the aforementioned family of polynomials are just a natural extension of those of the ordinary case.

A natural further extension is obtained by considering the following case of neo-Hermite polynomials of order $m$ :

$$
f\left(x t+y t^{m}\right)=\sum_{n=0}^{\infty} \frac{t^{n}}{n !} \phi_{n}^{(m)}(x, y),
$$

where

$$
\phi_{n}^{(m)}(x, y):=n ! \sum_{s=0}^{[n / m]} \frac{f_{n-(m-1) s} x^{n-m s} y^{s}}{(n-m s) ! s !} .
$$

When $f$ in the definition (2.13) is replaced by the exponential function, these neo-Hermite polynomials of order $m$ precisely coincide with the Gould-Hoper polynomials $g_{n}^{m}(x, y)$, defined by (see, e.g., [9, Section 1.11, equation (27)]; see also [8])

$$
\exp \left(x t+y t^{m}\right)=\sum_{n=0}^{\infty} \frac{t^{n}}{n !} g_{n}^{m}(x, y),
$$

so that, explicitly, we have [9, Section 1.9, equation (6)]

$$
\begin{aligned}
g_{n}^{m}(x, y) & =\sum_{k=0}^{[n / m]} \frac{n !}{k !(n-m k) !} x^{n-m k} y^{k} \\
& =x^{n}{ }_{m} F_{0}\left[-\frac{n}{m},-\frac{n-1}{m}, \ldots,-\frac{n-m+1}{m} ;-;\left(-\frac{m}{x}\right)^{m} y\right]
\end{aligned}
$$

in terms of a generalized hypergeometric function. 
It is fairly simple to prove the following recurrences relations:

$$
\begin{gathered}
\hat{f}^{-1} \frac{\partial}{\partial x} \phi_{n}^{(m)}(x, y)=n \phi_{n-1}^{(m)}(x, y) \\
{\left[(\hat{f} x)+m y \hat{f}^{-(m-1)} \frac{\partial^{m-1}}{\partial x^{m-1}}\right] \phi_{n}^{m}(x, y)=\phi_{n+1}^{m}(x, y),}
\end{gathered}
$$

as well as the associated differential equation

$$
\left[x \frac{\partial}{\partial x}+m y \hat{f}^{-(m-1)} \frac{\partial^{m}}{\partial x^{m}}\right] \phi_{n}^{m}(x, y)=n \phi_{n}^{m}(x, y)
$$

These last results, (2.17) and (2.18), complete the goals of the present section. Further comments on possible generalizations and deeper insight into their theory will be presented in the following section.

3. Further extensions and Bessel-type equations. In Section 2, we have seen the possibility of using a formalism close to that of the ordinary HKdF polynomials to treat the polynomials associated with the Taylor expansion of a composite function. To take a step forward, we consider the following threevariable case:

$$
f\left(x t+y t^{2}+z t^{3}\right)=\sum_{n=0}^{\infty} \frac{t^{n}}{n !} \phi_{n}^{(3)}(x, y, z),
$$

which readily yields

$$
\phi_{n}^{(3)}(x, y, z)=n ! \sum_{r=0}^{[n / 3]} \frac{(\hat{f} z)^{r} \phi_{n-3 r}(x, y)}{(n-3 r) ! r !}
$$

It is clear that it can be appropriately extended to a larger number of variables.

It is easily observed from the generating function (1.1) that

$$
\left(\frac{d}{d t}\right)^{l} f\left(x t+y t^{2}\right)=\sum_{n=0}^{\infty} \frac{t^{n}}{n !} \phi_{n+l}(x, y) .
$$

The so-called Rainville-type generating function occurring on the right-hand side of (3.3) can be easily found as follows (see the appendix):

$$
\sum_{n=0}^{\infty} \frac{t^{n}}{n !} \phi_{n+l}(x, y)=\exp \left[(\hat{f} x t)+\left(\hat{f} y t^{2}\right)\right] \phi_{l}(x+2 t y, y)
$$

The result (3.4) can be extended to the $m$-variable case, but this aspect of the problem and its connection with the Faà di Bruno formula for the derivative of composite functions will be discussed in a forthcoming publication. 
Before concluding this section, we will discuss the extension of the present results to the generalizations of Bessel-type functions. We consider the generating function:

$$
f(i x \sin \vartheta)=\sum_{n=-\infty}^{\infty} \exp (\text { in } \vartheta) \mathscr{B}_{n}(x),
$$

where

$$
\mathscr{B}_{n}(x)=\sum_{r=0}^{\infty} \frac{(-1)^{r} f_{n+2 r}}{r !(n+r) !}\left(\frac{x}{2}\right)^{n+2 r} .
$$

In general, the function $\mathscr{B}_{n}(x)$ is given by the integral representation

$$
\mathscr{B}_{n}(x)=\frac{1}{2 \pi} \int_{0}^{2 \pi} f(i x \sin \vartheta) \exp (-i n \vartheta) d \vartheta .
$$

The use of the formalism, which we have developed so far, allows us to transform the integral (3.7) into a more compact form. Indeed, by defining

$$
\begin{gathered}
\mathscr{C}(\hat{f} x):=\frac{f(i x)+f(-i x)}{2}, \\
\mathscr{S}(\hat{f} x):=\frac{f(i x)-f(-i x)}{2 i},
\end{gathered}
$$

we note that, from a formal point of view, we have

$$
\mathscr{C}[(\hat{f} x)+(\hat{f} y)]=\mathscr{C}[(\hat{f} x)] \mathscr{C}[(\hat{f} y)]-\mathscr{Y}[(\hat{f} x)] \mathscr{S}[(\hat{f} y)]
$$

and analogously for the sine-like part. Furthermore, since

$$
f(i x) \exp (i y)=\exp [i((\hat{f} x)+y)]
$$

we can conclude that

$$
\mathscr{B}_{n}(x)=\frac{1}{\pi} \int_{0}^{\pi} \mathscr{C}[(\hat{f} x \sin (\vartheta))-n \vartheta] d \vartheta .
$$

It is important to emphasize here that the functions $\mathscr{B}_{n}(x)$ satisfy recurrence relations of the following types:

$$
\begin{aligned}
\hat{f}^{-1} \frac{d}{d x} \mathscr{\Re}_{n}(x) & =\frac{1}{2}\left[\mathscr{B}_{n-1}(x)-\mathscr{B}_{n+1}(x)\right], \\
\frac{n \hat{f}^{-1}}{x} \mathscr{B}_{n}(x) & =\frac{1}{2}\left[\mathscr{B}_{n-1}(x)+\mathscr{B}_{n+1}(x)\right],
\end{aligned}
$$

which can be combined to obtain the following differential equation:

$$
\left[x^{2} \frac{d^{2}}{d x^{2}}+x \frac{d}{d x}-\left(n^{2}-\hat{f}^{2} x^{2}\right)\right] \mathscr{B}_{n}(x)=0 .
$$


Equation (3.13) immediately reduces to the ordinary Bessel equation when $\hat{f}=$ 1.

For a further form of Bessel-like functions, we consider the following generating function:

$$
f\left(i x \sin \vartheta-y(\sin \vartheta)^{2}\right)=\sum_{n=-\infty}^{\infty} \exp (i n \vartheta)_{\phi} \mathscr{S}_{n}(x, y)
$$

where

$$
{ }_{\phi} \mathscr{B}_{n}(x, y)=\sum_{r=0}^{\infty} \frac{(-1)^{r} \phi_{n+2 r}(x, y)}{2^{n+2 r} r !(n+r) !}
$$

The functions ${ }_{\phi} \mathscr{B}_{n}(x, y)$ play the same role as the Hermite-Bessel functions discussed in [4] and will be more thoroughly discussed in a forthcoming investigation.

4. Remarks and observations. In the previous sections, we have considered polynomials which can be viewed as generalized forms of the HKdF polynomials. Just to give an example of analogous work involving other polynomials, we consider here the following extension of the simple Laguerre family:

$$
(1-y t)^{-1} f\left(-\frac{x t}{1-y t}\right)=\sum_{n=0}^{\infty} t^{n} l_{n}(x, y) \quad(|y t|<1) .
$$

According to the already developed formalism, we can see that

$$
l_{n}(x, y)=n ! \sum_{r=0}^{n} \frac{(-1)^{r} f_{r} x^{r} y^{n-r}}{(n-r) !(r !)^{2}}
$$

We can derive, from the explicit representation (4.2), the relevant recurrence relations as follows:

$$
\begin{aligned}
\left(y-\hat{f} \hat{D}_{x}^{-1}\right) l_{n}(x, y) & =l_{n+1}(x, y), \\
-\hat{f}^{-1} \frac{\partial}{\partial x} x \frac{\partial}{\partial x} l_{n}(x, y) & =n l_{n-1}(x, y),
\end{aligned}
$$

where $\hat{D}_{x}^{-1}$ denotes the inverse of the derivative operator. A combination of (4.3) provides the differential equation

$$
\left[y \hat{f}^{-1} x \frac{\partial^{2}}{\partial x^{2}}+\left(y \hat{f}^{-1}-x\right) \frac{\partial}{\partial x}+n\right] \ln (x, y)=0
$$


Finally, we note that for this family of polynomials too, we have identities of the type (2.10), which lead us to

$$
\frac{\partial}{\partial y} l_{n}(x, y)=-\hat{f}^{-1} \frac{\partial}{\partial x} x \frac{\partial}{\partial x} l_{n}(x, y)
$$

with

$$
l_{n}(x, 0)=\frac{(-x)^{n}}{n !} f_{n}
$$

Before concluding this paper, we discuss a few applications of the above results.

For the evaluation of the following integral:

$$
I_{n}(x, y ; \alpha)=\frac{1}{2 \pi} \int_{0}^{2 \pi}\left(1+i x \sin \vartheta-y(\sin \vartheta)^{2}\right)^{\alpha} \exp (-i n \vartheta) d \vartheta
$$

it is evident that, according to (3.14), we have

$$
I_{n}(x, y ; \alpha)={ }_{\phi} \mathscr{B}_{n}(x, y)
$$

for

$$
f_{n}=\frac{\Gamma(\alpha+1)}{\Gamma(\alpha-n+1)}
$$

Similarly, we find that

$$
\frac{1}{2 \pi} \int_{0}^{2 \pi} \frac{e^{-i n \vartheta}}{1+i x \sin \vartheta} d \vartheta=\mathscr{B}_{n}(x)
$$

for

$$
f_{n}=n !
$$

We now consider the evaluation of the $l$ th derivative of the function

$$
\left(1+\frac{\sin (\sqrt{x})}{\sqrt{x}}\right)^{\alpha} .
$$

By using the known approximation [3]:

$$
\begin{gathered}
\frac{\sin x}{x}=1+a x^{2}+b x^{4}+\varepsilon(x), \\
\left(|\varepsilon(x)|<2 \cdot 10^{-4} ; a=-0.16605 ; b=0.00761 ; 0<x<\frac{\pi}{2}\right),
\end{gathered}
$$


we find that

$$
\left(1+\frac{\sin (\sqrt{x})}{\sqrt{x}}\right)^{\alpha} \simeq 2^{\alpha} \sum_{n=0}^{\infty} \frac{x^{n}}{n !} \phi_{n}\left(\frac{a}{2}, \frac{b}{2}\right)
$$

for $f_{n}$ given by (4.9).

The $l$ th derivative can now be evaluated by means of (3.3). Better precision can be achieved by considering more terms in (4.13) and by using higher-order polynomials of the type (3.2). This aspect of the problem will be discussed in a forthcoming investigation where we will enter more deeply into the theory of the multivariable polynomials $\phi_{n}(x, y, z, \ldots)$ and the theory of the Bessel functions, and will also discuss their connections with several previously obtained results.

Appendix. The proof of the Rainville-type generating function (3.4) follows form the fact that

$$
\left[(\hat{f} x)+2 y \frac{\partial}{\partial x}\right]^{n} \phi_{l}(x, y)=\phi_{n+l}(x, y) .
$$

Multiplying both sides of (A.1) by $t^{n} / n$ ! and then summing over $n$, we find that

$$
\sum_{n=0}^{\infty} \frac{t^{n}}{n !} \phi_{n+l}(x, y)=\exp \left[(\hat{f} x t)+2 y t \frac{\partial}{\partial x}\right] \phi_{l}(x, y)
$$

According to (2.9), we have

$$
\sum_{n=0}^{\infty} \frac{t^{n}}{n !} \phi_{n+l}(x, y)=\exp \left[(\hat{f} x t)+\left(\hat{f} y t^{2}\right)\right] \cdot \exp \left[2 y t \frac{\partial}{\partial x}\right] \phi_{l}(x, y)
$$

which immediately yields (3.4).

ACKNOWLEDGment. The present investigation was supported, in part, by the Natural Sciences and Engineering Research Council of Canada under Grant OGP0007353.

\section{REFERENCES}

[1] P. Appell and J. Kampé de Fériet, Fonctions Hypergéométriques et Hypersphériques; Polynômes d'Hermite, Gauthier-Villars, Paris, 1926 (French).

[2] R. P. Boas Jr. and R. C. Buck, Polynomial Expansions of Analytic Functions, SpringerVerlag, Berlin, Göttingen, and Heidelberg, 1958, (reprinted (with corrections) by Academic Press, New York and Springer-Verlag, Berlin, 1964).

[3] B. Carlson and M. Goldstein, Rational Approximation of Functions, Los Alamos Scientific Laboratory, New Mexico, 1955. 
[4] G. Dattoli, Hermite-Bessel and Laguerre-Bessel functions: a by-product of the monomiality principle, Advanced Special Functions and Applications (Melfi, 1999) (D. Cocolicchio, G. Dattoli, and H. M. Srivastava, eds.), Proc. Melfi Sch. Adv. Top. Math. Phys., vol. 1, Rome, 2000, pp. 147-164.

[5] G. Dattoli, S. Lorenzutta, and D. Sacchetti, A note on operational rules for Hermite and Laguerre polynomials: operational rules and special polynomials, Int. J. Math. Stat. Sci. 9 (2000), no. 2, 227-238.

[6] G. Dattoli, P. L. Ottaviani, A. Torre, and L. Vázquez, Evolution operator equations: integration with algebraic and finite-difference methods. Applications to physical problems in classical and quantum mechanics and quantum field theory, Riv. Nuovo Cimento Soc. Ital. Fis. (4) 20 (1997), no. 2, 1-133.

[7] G. Dattoli, P. E. Ricci, and C. Cesarano, A note on Legendre polynomials, Int. J. Nonlinear Sci. Numer. Simul. 2 (2001), no. 4, 365-370.

[8] H. W. Gould and A. T. Hopper, Operational formulas connected with two generalizations of Hermite polynomials, Duke Math. J. 29 (1962), 51-63.

[9] H. M. Srivastava and H. L. Manocha, A Treatise on Generating Functions, Ellis Horwood Series: Mathematics and Its Applications, John Wiley \& Sons, New York, 1984.

G. Dattoli: Unita Tecnico Scientificà Tecnologie Fisiche, Gruppo Fisica Teorica e Matematica Applicata, Ente Nationale per le Nuove Tecnologie, l'Energia e l'Ambiente, Via Enrico Fermi 45, 00044 Frascati, Roma, Italy

E-mail address: dattoli@frascati .enea.it

H. M. Srivastava: Department of Mathematics and Statistics, University of Victoria, Victoria, British Columbia, Canada V8W 3P4

E-mail address: harimsri@math.uvic.ca

D. Sacchetti: Dipartimento di Statistica, Probabilitá e Statistiche Applicate, Università degli Studi di Roma “La Sapienza," Piazzale Aldo Moro 5, 00185 Roma, Italy

E-mail address: dario.sacchetti@uni roma1.it 


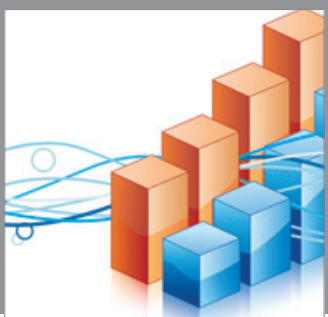

Advances in

Operations Research

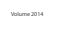

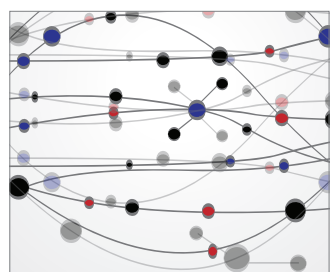

\section{The Scientific} World Journal
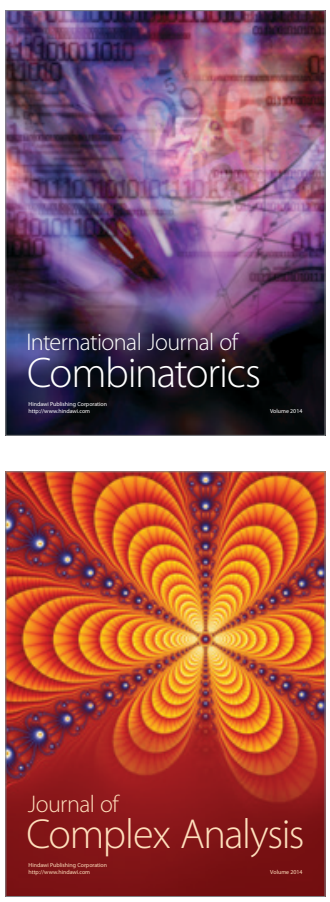

International Journal of

Mathematics and

Mathematical

Sciences
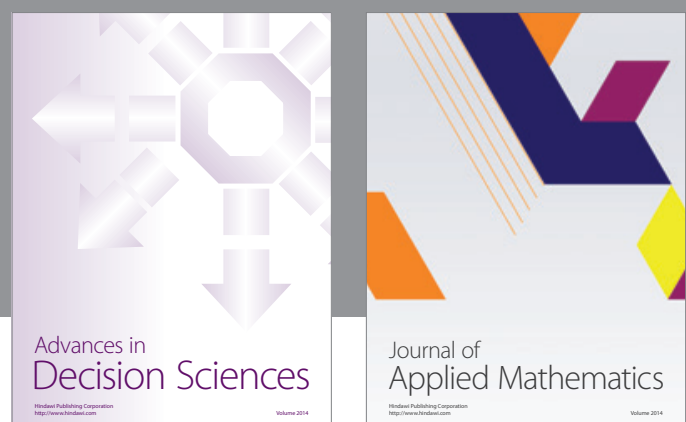

Journal of

Applied Mathematics
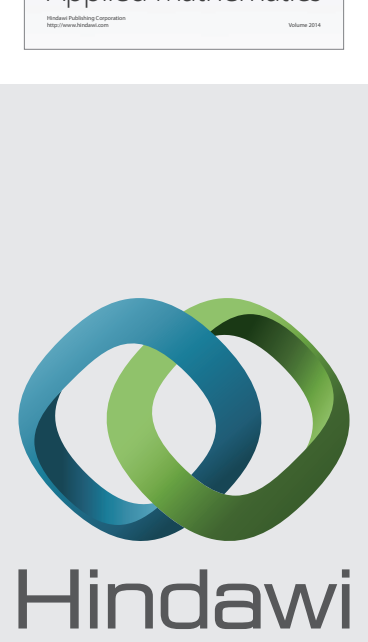

Submit your manuscripts at http://www.hindawi.com
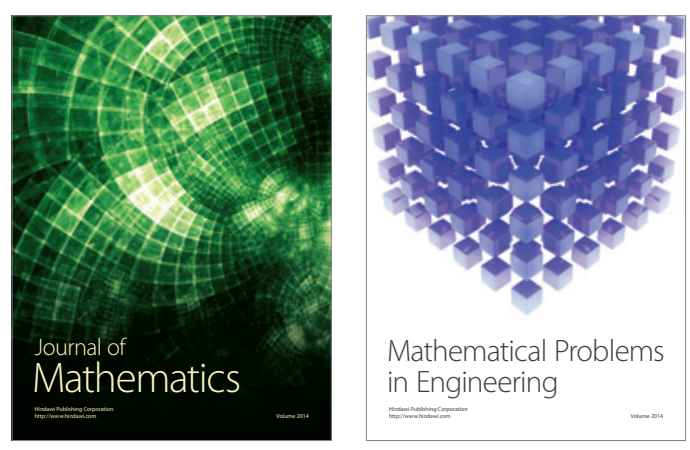

Mathematical Problems in Engineering
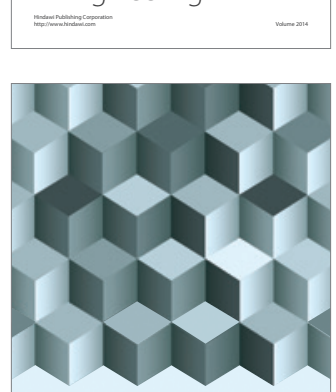

Journal of

Function Spaces
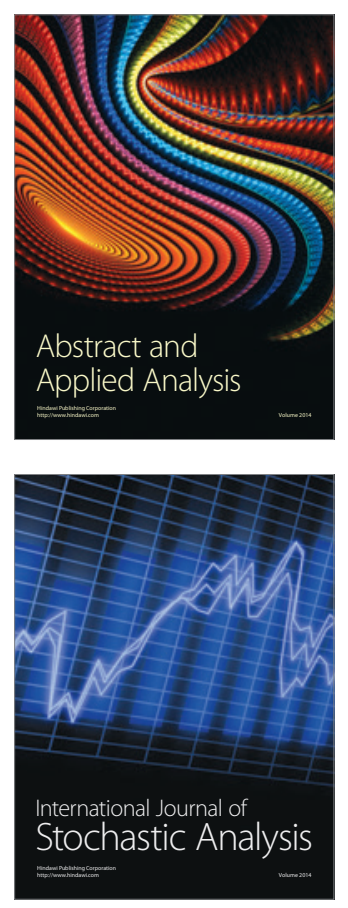

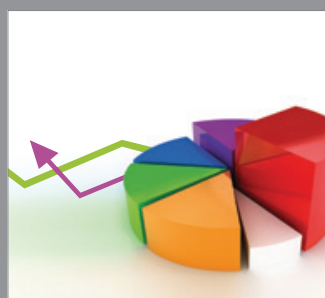

ournal of

Probability and Statistics

Promensencen
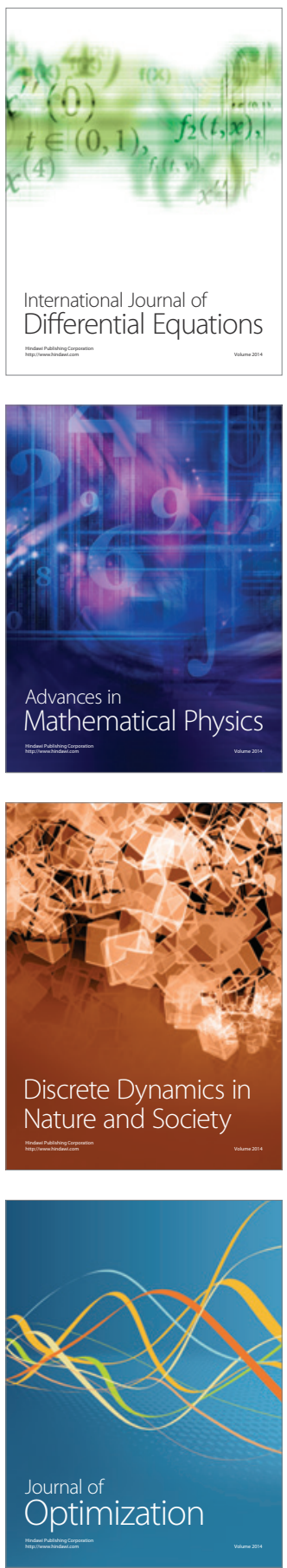